\title{
Objective optical quality in eyes with customized selection of aspheric intraocular lens implantation
}

\author{
Qing-Qing $\operatorname{Tan}^{1,2+} \mathbb{D}$, Jia Lin ${ }^{1,2+}$, Jing $\operatorname{Tian}^{1,2}$, Xuan Liao ${ }^{1,2^{*}}$ and Chang-Jun $\operatorname{Lan}^{1,2^{*}}$ (D)
}

\begin{abstract}
Background: To compare the postoperative optical quality in eyes with customized selection and random selection of aspheric intraocular lens (IOL) implantation.

Methods: A prospective, nonrandomized study was implemented in adult cataract patients who underwent unilateral phacoemulsification with aspheric IOL implantation. Patients were allocated into two treatment groups: a customized group and a control group. In the customized group, the aspheric IOL selection was based on the corneal spherical aberration to enable the postoperative target ocular spherical aberration closest to zero; in the control group, the aspheric IOLs were chosen using a random strategy. Primary outcome measurements included the following objective optical quality assessments: higher-order aberrations obtained by a Hartmann-shack aberrometer at $4 \mathrm{~mm}$ and $6 \mathrm{~mm}$ pupil diameters; objective scatter index (OSI), modulation transfer function (MTF) cut-off, Strehl ratio (SR) and a simulated contrast visual acuity_optical quality analysis system value (OV) obtained by a double-pass system with a 4-mm aperture. Subjective visual acuity was measured as secondary outcome. All the patients were followed up for 3 months.
\end{abstract}

Results: Eighty-four patients in the customized group and 78 patients in the control group were evaluated. There was no significant difference in postoperative visual acuity between the two groups $(P>0.05)$. Significantly less ocular higher-order aberrations were shown in the customized group $(P<0.05)$. No significant difference was shown in OSI, MTF cut-off, SR and OV between the two groups $(P>0.05)$.

Conclusions: Although customized selection of aspheric $\mathrm{IOL}$ implantation showed less postoperative ocular aberrations, it performed similarly to random selection of aspheric IOL implantation in terms of postoperative visual acuity, simulated contrast visual acuity, intraocular scatter, modulation transfer function and Strehl ratio.

Trial registration: Retrospectively registered on 07/06/2019. Registration number: ChiCTR1900024356.

Keywords: Cataract, Aspheric IOL, Customized, Higher-order aberrations, OQAS

\section{Background}

To obtain optimal optical quality and visual performance for the pseudophakic eyes, reducing the higher-order aberrations (HOAs) is demanded. As one of the most important HOAs, spherical aberration (SA) has been paid much attention by researchers [1]. A human cornea has a SA of $+0.27 \mu \mathrm{m}$ as the mean value at $6 \mathrm{~mm}$ pupil

\footnotetext{
*Correspondence: aleexand@163.com; eyelanchangjun@163.com ${ }^{+}$Qing-Qing Tan and Jia Lin contributed equally to this work.

'Department of Ophthalmology, Affiliated Hospital of North Sichuan Medical College, 1 Mao Yuan Nan Road, Nanchong 637000, Sichuan, China Full list of author information is available at the end of the article
}

diameter (PD) [2], a spherical intraocular lens (IOL) has positive SA as well, thus it could worsen the postoperative ocular SA and then impair the retinal image quality; an aspheric IOL has a prolate aspheric surface that creates a zero or negative value of SA, which could totally or partially compensate the corneal positive SA, then reduce the postoperative ocular SA and therefore improve the visual performance [3]. Currently, there are several clinically available aspheric IOLs with different labeled SA values, such as the SA value of $-0.27 \mu \mathrm{m}$ (AMO Tecnis ZCB00), $-0.20 \mu \mathrm{m}$ (Alcon SN60WF IQ) and $0 \mu \mathrm{m}$ (Bausch \& Lomb Akreos Adapt AO). These

(c) The Author(s). 2019 Open Access This article is distributed under the terms of the Creative Commons Attribution 4.0 International License (http://creativecommons.org/licenses/by/4.0/), which permits unrestricted use, distribution, and 
different labeled SA designs aim to achieve optimal optical quality and visual performance by appropriately compensating preoperative corneal SA.

Wavefront aberrometers have been widely used in previous studies for assessing the optical quality by measuring the aberrations. However, they have a lack of information on quite higher-order aberrations and intraocular scatter [4]. In addition to aberrations, intraocular scatter is another important optical factor affecting visual performance. Optical quality analysis system (OQAS), based on the double-pass technique, provides information for both aberrations and intraocular scatter and produces a more accurate and complete description of the optical quality $[4,5]$. OQAS provides parameters including objective scatter index (OSI), modulation transfer function (MTF) cut-off, Strehl ratio (SR), and a simulated contrast visual acuity-optical quality analysis system values (OV) at contrasts of 100, 20 and 9\%, which allow comprehensive assessment of the optical quality of the human eyes [6]. It has been successfully used to evaluate ocular optical quality in pseudophakic eyes $[7,8]$ and exhibited good repeatability $[9,10]$.

Previous non-controlled studies reported that applying a customized selection of aspheric IOL implantation based on corneal SA could precisely reduce the postoperative ocular SA closest to zero [11, 12]. However, the current limited controlled studies merely measured SA or contrast sensitivity for assessing the visual quality, and there is still a lack of consensus whether optical quality following a customized selection with a target ocular SA of zero differs from that following a non-customized selection $[13,14]$. In this study, we applied a random selection strategy in the control group, by which we aimed to replicate the aspheric IOL selection strategy that we have been using in actual clinical practice in the past. We hypothesized that customized selection creates an equal postoperative optical quality with a random selection of aspheric IOL implantation. Using a combination of Hartmann-shack aberrometer and double-pass instrument with a comprehensive and objective assessment, this study aimed to determine whether customized selection differs from random selection of aspheric IOL in postoperative optical quality.

\section{Methods}

\section{Study design and participants}

This prospective study was approved by the Institutional Review Board of Affiliated Hospital of North Sichuan Medical College. Adult cataract patients undergone unilateral phacoemulsification and IOL implantation from October 2015 to December 2016 in Affiliated Hospital of North Sichuan Medical College were enrolled. Informed consents were obtained from all patients. Exclusion criteria included corneal astigmatism>
1.00 diopters (D), irregular astigmatism and other ocular pathology that could potentially affect the postoperative vision, such as corneal pathology, uncontrolled glaucoma, existing retinal or optic neuropathy.

Totally 182 eyes of 182 patients were allocated into 2 treatment groups: the customized group and the control group. In the customized group, 92 eyes of 92 patients were implanted with an aspheric IOL selected based on corneal SA at $6 \mathrm{~mm}$ PD. The IOL allocation method in the customized group was adapted from Solomon's study in 2010 [11]. For corneal SA $<+0.1 \mu \mathrm{m}$, Bausch \& Lomb Akreos Adapt AO (with a labelled SA of $0 \mu \mathrm{m}$ ) was selected; for corneal $\mathrm{SA} \geq+0.1 \mu \mathrm{m}$ but $\leq+0.235 \mu \mathrm{m}$, Alcon SN60WF IQ was selected (with a labelled SA of $-0.20 \mu \mathrm{m}$ ); for corneal $\mathrm{SA}>+0.235 \mu \mathrm{m}$, AMO Tecnis ZCB00 was selected (with a labelled SA of $-0.27 \mu \mathrm{m}$ ). In the control group, 90 eyes of 90 patients were implanted with 1 of 3 aspheric IOL types stated above using a random selection strategy.

\section{Surgical procedures}

All operations were performed by the same experienced surgeon (CJL) and using the same phacoemulsification machine (Stellaris, Bausch \& Lomb Co. Ltd., USA). A $2.8 \mathrm{~mm}$ clear corneal incision at the meridian of $135^{\circ}$ was made, a continuous circular capsulorhexis was performed, followed by hydrodissection and phacoemulsification cataract extraction. Then, an aspheric IOL was implanted in the capsular bag.

\section{Outcome evaluation}

All patients had a comprehensive ophthalmic examination before the surgery. The irregularities of the cornea and the IOL allocation in the customized group were determined by measuring the corneal topography and wavefront aberrations using a Hartmann-shack based wavefront analyzer (KR-1 W, Topcon Corp., Tokyo, Japan).

All postoperative evaluations were performed at 3 months after surgery, including routine examinations: refraction, uncorrected visual acuity (UCVA), best corrected visual acuity (BCVA), slit-lamp examination, funduscopy, and tonometry. Visual acuity was measured under photopic condition $\left(85 \mathrm{~cd} / \mathrm{m}^{2}\right)$. Tilt and decentration of the IOLs in relation to the visual axis were assessed using a Scheimpflug imaging system (Pentacam, Oculus Inc., Wetzlar, Germany).

Meanwhile, wavefront aberrations were measured using the identical device as baseline measurements under mesopic condition $\left(3 \mathrm{~cd} / \mathrm{m}^{2}\right)$. Aberrations were measured after dilation by instillation of a mixture of 0.5\% tropicamide and 0.5\% phenylephrine (Mydrin P, Santen Pharmaceutical Co., Osaka, Japan) in all eyes. Based on Zernike coefficients, $\mathrm{SA}\left(\mathrm{Z}_{4}{ }^{0}\right)$, root mean square (RMS) values of coma $\left(\mathrm{Z}_{3}{ }^{-1}, \mathrm{Z}_{3}{ }^{1}\right)$, trefoil $\left(\mathrm{Z}_{3}{ }^{-3}\right.$, 
$\mathrm{Z}_{3}{ }^{3}$ ) and total higher-order aberration (t-HOA, 3rd to 6 th order aberrations) were analyzed for $4 \mathrm{~mm}$ PD and $6 \mathrm{~mm}$ PD respectively.

OQAS (OQAS II; Visiometrics SL, Terrassa, Spain) measurements were performed postoperatively in the same mesopic condition. All measurements were performed with a 4-mm aperture, which is a standard size used in clinical double-pass studies [15]. The following parameters were measured to quantify the optical quality: 1) OSI, defined as the ratio of the light of peripherally annular area versus that of the central peak in the acquired double-pass image, quantifies intraocular scatter [16]. The lower OSI values indicate better optical quality; 2) MTF cut-off, the cut-off point of the MTF curve on the $\mathrm{x}$-axis that can be directly computed from the point spread function (PSF), denotes the highest spatial frequency at which the MTF reaches the lowest contrast of $1 \%$ [16]. The higher MTF cut-off values indicate better optical quality; 3) SR, the ratio of peak focal intensities in aberrated PSF and ideal PSF, indicates a perfect optical system when it is equal to 1 and an aberrated optical system when it is less than 1 [16]; 4) OVs (OV 100\%, OV 20\% and OV 9\%), corresponding to three different spatial frequencies of the MTF values, describe optical quality for three contrast conditions that are commonly used in ophthalmology practice [16]. Specifically, OV $100 \%$ is directly related to the MTF cut-off frequency [17]. The OVs can be used to obtain more specific information about the behavior of the system at different contrasts, which may remain hidden when more global parameters that integrate the information along all available spatial frequencies are considered, such as the Strehl ratio [18].

\section{Statistical analysis}

All data were analyzed using SPSS 20.0 software. A Shapiro-Wilk test was used for testing the normal distribution. Normal distributions were shown in all continuous data in both groups $(P>0.05)$. Continuous variables were expressed as Mean \pm standard deviation (SD), dichotomous variables were expressed as percent $(\mathrm{n} \%)$. An independent samples $t$-test was performed for comparisons between the two groups, and a contingency Chi-square test was performed for dichotomous data. A $P$ value $<0.05$ was considered statistically significant.

\section{Results}

Eighty-four patients (38 women, 46 men) in the customized group and 78 patients (37 women, $41 \mathrm{men}$ ) in the control group were evaluated. The mean age was $65.26 \pm 8.91$ years old in the customized group and $67.84 \pm 9.64$ years old in the control group. Preoperative demographics, corneal astigmatism, corneal HOAs and IOL power showed no significant difference between the two groups (Table 1). In the customized group, Akreos Adapt AO IOLs were selected for 1 patient, whose preoperative corneal SA was $0.085 \mu \mathrm{m}$; SN60WF IQ IOLs were selected for 26 patients, whose mean preoperative corneal SA was $0.21 \pm 0.05 \mu \mathrm{m}$; Tecnis ZCB00 IOLs were selected for 57 patients, whose mean preoperative corneal SA was $0.33 \pm 0.08 \mu \mathrm{m}$. In the control group, Akreos Adapt AO, SN60WF IQ and Tecnis ZCB00 IOLs were selected for 27, 26 and 25 patients respectively. There was no intraoperative complication in any case. During follow-up, no tilt $>4^{\circ}$ or decentration $>0.25 \mathrm{~mm}$ of any IOL was encountered, which were significantly lower than the clinically significant tilt and decentration cutoffs $\left(7^{\circ}\right.$ and $0.4 \mathrm{~mm}$, respectively) defined by Holladay et al. [19]. The differences in tilt and decentration between the two groups were not statistically significant $(P>0.05$; independent $t$-test). The following patients were excluded: in the customized group, 1 patient had severe posterior capsular opacity, 1 patient had macular edema and 6 patients were lost to follow-up; in the control group, 2 patient had severe posterior capsular opacity, 10 patients were lost to follow-up.

Table 1 Demographics and baseline characteristics of the patients

\begin{tabular}{llll}
\hline & Customized group $N=84$ & Control group $N=78$ & $P$ value \\
\hline Sex (female, \%) & $45.2 \%$ & $47.4 \%$ & $0.78^{\mathrm{a}}$ \\
Laterality (surgery in the right eye, \%) & $46.4 \%$ & $55.1 \%$ & $0.27^{\mathrm{a}}$ \\
Age (Mean \pm SD, year) & $65.26 \pm 8.91$ & $67.84 \pm 9.64$ & $0.07^{\mathrm{b}}$ \\
Corneal astigmatism (Mean \pm SD, D) & $0.59 \pm 0.21$ & $0.52 \pm 0.25$ & $0.07^{\mathrm{b}}$ \\
IOL power (Mean \pm SD, D) & $19.91 \pm 3.43$ & $19.42 \pm 3.36$ & $0.36^{\mathrm{b}}$ \\
t-HOA (6 mm pupil) & $0.69 \pm 0.19$ & $0.75 \pm 0.25$ & $0.15^{\mathrm{b}}$ \\
SA (6 mm pupil) & $0.28 \pm 0.07$ & $0.29 \pm 0.07$ & $0.66^{\mathrm{b}}$ \\
Coma (6 mm pupil) & $0.21 \pm 0.10$ & $0.23 \pm 0.13$ & $0.38^{\mathrm{b}}$ \\
Trefoil (6 mm pupil) & $0.49 \pm 0.18$ & $0.52 \pm 0.22$ & $0.36^{\mathrm{b}}$ \\
\hline
\end{tabular}

$\overline{t-H O A}$ total higher-order aberration, $S A$ spherical aberration, $S D$ standard deviation, $D$ diopter; ${ }^{a}$ : Chi-square test; ${ }^{\mathrm{b}}:$ Independent $\mathrm{t}$-test 


\section{Visual acuity}

There was no significant difference in postoperative UCVA $(0.14 \pm 0.16$ LogMAR for the customized group vs $0.16 \pm 0.14 \log M A R$ for the control group; $P=0.4$; independent $t$-test) and BCVA $(0.03 \pm 0.07 \operatorname{LogMAR}$ for the customized group vs $0.05 \pm 0.07 \operatorname{LogMAR}$ for the control group; $P=0.19$; independent $t$-test) between the two groups.

\section{Wavefront aberrations}

There was no significant difference in corneal SA before and after surgery at both $4 \mathrm{~mm}$ PD (pre, $0.06 \pm 0.02 \mu \mathrm{m}$; post, $0.05 \pm 0.05 \mu \mathrm{m} ; P=0.28$; paired $t$-test) and $6 \mathrm{~mm}$ PD (pre, $0.28 \pm 0.09 \mu \mathrm{m}$; post, $0.28 \pm 0.07 \mu \mathrm{m} ; P=0.80$; paired $t$-test) for the entire study population. Table 2 shows the postoperative ocular aberration comparisons between the two groups. As expected, all measured HOAs increased when pupil increased from $4 \mathrm{~mm}$ to 6 $\mathrm{mm}$. At $4 \mathrm{~mm}$ PD, postoperative ocular SA was significantly lower in the customized group than in the control group $(0.00 \pm 0.03 \mu \mathrm{m}$ vs $0.02 \pm 0.05 \mu \mathrm{m}$, respectively; $P=0.003$ ), while no significant difference was shown in $\mathrm{t}$-HOA, coma and trefoil $(P>0.05)$; at $6 \mathrm{~mm} \mathrm{PD}$, significantly lower $\mathrm{t}-\mathrm{HOA}(0.66 \pm 0.17 \mu \mathrm{m}$ vs $0.74 \pm 0.16 \mu \mathrm{m} ; P<$ $0.001)$, SA $(0.00 \pm 0.12 \mu \mathrm{m}$ vs $0.12 \pm 0.18 \mu \mathrm{m} ; P<0.001)$, coma $(0.25 \pm 0.13 \mu \mathrm{m}$ vs $0.33 \pm 0.16 \mu \mathrm{m} ; P=0.002)$ and trefoil $(0.56 \pm 0.16 \mu \mathrm{m}$ vs $0.62 \pm 0.16 \mu \mathrm{m} ; P=0.019)$ were shown in the customized group than in the control group. Moreover, in the customized group, 81\% (68/84) of the eyes achieved an ocular SA less than or equal to $0.15 \mu \mathrm{m}$ postoperatively.

\section{OQAS parameters}

Table 3 shows the OQAS parameter comparisons between the two groups. Customized group demonstrated slightly higher MTF cut-off, OV100\%, OV20\%, OV9\%, and lower OSI than the control group with a 4-mm aperture, however, the differences between the two groups were not statistically significant $(P=0.54, P=$ $0.65, P=0.73, P=0.64$, and $P=0.84$, respectively). The

Table 2 Postoperative wavefront aberrations (Mean \pm SD, $\mu \mathrm{m}$ )

\begin{tabular}{lllll}
\hline Ocular aberrations & Customized group & Control group & $P$ value \\
\hline $4 \mathrm{~mm}$ & $\mathrm{t}-\mathrm{HOA}$ & $0.20 \pm 0.06$ & $0.21 \pm 0.06$ & 0.050 \\
& SA & $0.00 \pm 0.03$ & $0.02 \pm 0.05$ & $0.003^{\mathrm{a}}$ \\
& Coma & $0.09 \pm 0.05$ & $0.10 \pm 0.04$ & 0.066 \\
& Trefoil & $0.18 \pm 0.06$ & $0.20 \pm 0.06$ & 0.088 \\
$6 \mathrm{~mm}$ & $\mathrm{t}-\mathrm{HOA}$ & $0.66 \pm 0.17$ & $0.74 \pm 0.16$ & $<0.001^{\mathrm{a}}$ \\
& SA & $0.00 \pm 0.12$ & $0.12 \pm 0.18$ & $<0.001^{\mathrm{a}}$ \\
& Coma & $0.25 \pm 0.13$ & $0.33 \pm 0.16$ & $0.002^{\mathrm{a}}$ \\
& Trefoil & $0.56 \pm 0.16$ & $0.62 \pm 0.16$ & $0.019^{\mathrm{a}}$ \\
\hline
\end{tabular}

${ }^{a}$ : indicates statistical significance
Table 3 Postoperative OQAS parameters (Mean \pm SD)

\begin{tabular}{llll}
\hline OQAS parameters & Customized group & Control group & $P$ value \\
\hline OSI & $1.47 \pm 0.46$ & $1.50 \pm 0.71$ & 0.84 \\
MTF cut-off (c/deg) & $28.41 \pm 8.70$ & $27.50 \pm 9.17$ & 0.54 \\
SR & $0.15 \pm 0.04$ & $0.15 \pm 0.04$ & 1.00 \\
OV100\% & $0.95 \pm 0.29$ & $0.93 \pm 0.31$ & 0.65 \\
OV20\% & $0.64 \pm 0.21$ & $0.62 \pm 0.24$ & 0.73 \\
OV9\% & $0.36 \pm 0.13$ & $0.35 \pm 0.13$ & 0.64 \\
\hline
\end{tabular}

OSI objective scatter index, MTF modulation transfer function, SR Strehl ratio, OV OQAS value, c/deg. cycle per degree

two groups demonstrated the same in SR $(0.15 \pm 0.04$ in both groups; $P=1.00$ ).

\section{Discussion}

With the advance in wavefront technology, customized selection of aspheric IOLs to compensate individual corneal aberrations has been a trend in the current era of refractive cataract surgery. The aspheric IOLs are designed to compensate the positive corneal SA and thus reduce the ocular SA. The availability of various aspheric IOL designs has enabled the customized selection to precisely achieve a postoperative target ocular SA of zero, which has been considered to be the optimal SA value for producing the best optical quality and visual performance by some researchers [20, 21]. However, there are few controlled studies on optical quality of customized selection with a target SA of zero and the conclusions are inconsistent $[13,14]$. The present study aimed to comprehensively compare the postoperative optical quality using a combination of Hartmann-shack wavefront aberrometer and double-pass instrument between customized and random selections of aspheric IOLs, which has been rarely applied in previous studies regarding the customized selection with a target ocular SA of zero.

In this study, no significant differences in postoperative UCVA and BCVA were observed between the two groups, which were consistent with the findings of previous controlled studies related to customized selection $[13,14,22,23]$. The finding of the mean corneal SA at $6 \mathrm{~mm}$ PD was comparable to that in the study by Beiko et al. [2] $(0.28 \mu \mathrm{m}$ vs $0.27 \mu \mathrm{m})$, and the unchanged corneal SA after surgery indicated that a bias resulting from the surgical impacts on corneal SA could be avoided. With a $6 \mathrm{~mm}$ PD, t-HOA, SA, coma and trefoil were all significantly lower in the customized group, indicating that customized selection reduced the ocular aberrations more effectively than random selection. Moreover, in the customized group, the mean postoperative ocular SA was $0.00 \mu \mathrm{m}$, which indeed met the goal of this study, that was, rendering the postoperative ocular SA to be closest to zero. This result was also consistent with the 
previous studies $[11,12]$. In the control group, the postoperative ocular SA was $0.12 \pm 0.18 \mu \mathrm{m}$, indicating that almost half of the corneal SA remained by using a random selection, which was similar to the result in the study using the same selection strategy by Jia et al. [22] (a residual SA of $0.152 \pm 0.151 \mu \mathrm{m}$ ). As is well-known, the coma and trefoil aberrations are mainly related to IOL tilt, decentration and surgical incisions, and the IOL tilt and decentration differ among different types of IOLs [24]. Although the same incisional procedure was used and comparable tilt and decentration were presented in both groups, results demonstrated that coma and trefoil showed significant difference between the two groups when the pupil increased. The only controlled study on customized selection that reported coma and trefoil aberrations was by Nochez et al. [23], which compared a customized group targeting SA to + $0.1 \mu \mathrm{m}$ with a control group receiving zero SA IOLs only. Results showed similar coma and trefoil in both groups. However, as mentioned, the study design and the IOLs used by Nochez et al. were quite different from the present study, which might lead to the inconsistent results. The unexpected results in this study might be due to the difference of IOL selection strategies in the two groups, which led to imbalance in IOL allocation and thus SA distribution of IOLs between groups. IOLs with various SA values could induce various degree of coma, trefoil and thus t-HOA, even with less noticeable tilt or decentration $[16,25]$. However, we had a good reason to apply a random selection strategy as a control, which was the strategy we have been using in actual clinical practice in the past. Overall, the results regarding wavefront aberrations in this study suggested that customized selection of aspheric IOLs based on corneal SA is feasible to achieve a target ocular SA of zero, and a random selection lead to a positive residual SA of $0.12 \mu \mathrm{m}$. Customized selection demonstrated a better optical quality in terms of wavefront aberrations at 6 mm PD.

Although lower aberrations demonstrated in the customized group, similar values for all OQAS parameters were shown in both groups in the present study. The results for OVs that represent contrast visual acuity were consistent with Al-Sayyari et al. [13] who found no significant difference in contrast sensitivity between the customized group and the control group. The study by Nochez et al. [23] was the only study applying OQAS system to assess optical quality in customized selection, in which they found significantly better MTF cut-off, OV $100 \%$, OV $20 \%$ and OV $9 \%$ in the customized group than in the control group. However, their study design was quite different from the present study. They set up the target SA to $+0.1 \mu \mathrm{m}$ in the customized group and only two SA values $(0 \mu \mathrm{m}$ and $-0.18 \mu \mathrm{m})$ of aspheric
IOLs were available for selection. The control group received zero SA IOLs only. Eventually, they achieved a mean postoperative ocular SA of $0.085 \mu \mathrm{m}$ in the customized group and $0.261 \mu \mathrm{m}$ in the control group, the difference in the mean postoperative ocular SA between groups was $0.176 \mu \mathrm{m}$ in their study, which was much higher than that in the present study $(0.12 \mu \mathrm{m})$. This might be the main reason for the inconsistent results between the two studies. As was demonstrated in one of our previous studies, OQAS system could reveal inconsistent outcomes with aberrometers. In that study, we concluded that the optical quality might be overestimated when only aberrations were considered, thus combining the effect of ocular scattering with aberrations by an OQAS system would result in a more accurate assessment of optical quality [16]. Both aberrations and intraocular scatter are known to decrease the quality of the retinal image and therefore the visual performance. Previous evidence suggested that OSI had no significant correlation with SA in pseudophakic eyes $(r=0.133, P=0.525)$, whereas a significantly positive correlation with the total ocular aberrations $(r=0.451$, $P=0.024$ ) [26]. Although the $P$ value showed a statistically significance, the correlation coefficient was not large enough to suggest a clinical significance. This may explain why lower aberrations achieved by the customized selection did not lead to an OSI reduction compared to the random selection in the present study. Another previous study investigated the impacts of SA and intraocular scatter on contrast sensitivity, the results demonstrated that contrast sensitivity was reduced less by scatter when SA was present as compared with the cases without SA. They concluded that combined presence of positive SA and scatter could be a mild protective compensatory mechanism reducing the impact that the two factors may have on contrast sensitivity separately [27]. This viewpoint may explain why the overall optical quality interpreted by MTF cut-off, SR and OVs in the control group, though had a larger positive SA, were not worse than the customized group in this study.

In addition, there is currently controversy over the optimal target ocular SA values for an aspheric IOL: $0 \mu \mathrm{m}$ or $+0.10 \mu \mathrm{m}$. Some researchers argued that IOL implantation aiming to reach a target ocular SA to zero could lead to the best optical quality and visual performance [12, 20, 21, 28, 29]. Nevertheless, some researchers argued the same for creating a slightly positive residual ocular SA [22, 23, 30, 31]. Several controlled studies confirmed that both currently argued modes of target ocular SA provided desirable optical quality and visual performance. Specifically, zero SA was more beneficial in mesopic condition while slightly positive SA was more beneficial for near vision [14, 29]. One of the reasons why the overall optical quality of the customized selection 
did not differ from the random selection might also be that the mean residual ocular SA by a random selection in the present study $(+0.12 \mu \mathrm{m})$ was fairly comparable to $+0.1 \mu \mathrm{m}$, which was argued by previous studies to provide a comparable optical quality to that of a zero target ocular SA.

Moreover, as suggested by several previous studies: 1 ) although SA can be strongly reduced by aspheric IOLs, $\mathrm{t}-\mathrm{HOA}$ was only slightly reduced. This may be a reason for the unclear results in studies assessing the potential benefit to visual performance of aspheric IOLs [32]; 2) the optimal ocular SA producing the best image quality varied widely between eyes, which was highly associated with the amount of defocus. The amount of optimal ocular SA could be predicted based on other HOAs of the cornea as well. Selection of an aspherical IOL should be customized based on the full spectrum of corneal HOAs, not on SA alone [21]; 3) a study using adaptive optics vision simulator demonstrated that pseudophakic eyes experienced peak contrast sensitivity performance with varying levels of SA when their natural aberrations were present. However, average contrast performance peaked at SA of zero when all HOAs were corrected [20]. These studies indicated that interactions between SA with other HOAs and lower-order aberrations are complicated and not well understood. Customized selection of aspheric IOL implantation based on corneal SA alone may not be an appropriate approach to achieve optimal visual outcomes. Conclusions of these studies may also explain why there was no significant difference in the overall optical quality between customized and random selections of aspheric IOLs in the present study.

Compared with the previous controlled studies, the strengths of the present study were a larger sample size, a more specific customized selection with narrower SA ranges, as well as a more comprehensive and objective assessment for optical quality. There are also some limitations: 1) the focus of the customized design was only on the optimization of postoperative SA; 2) only one target ocular SA value $(0 \mu \mathrm{m})$ was set up for the customized selection; 3 ) subjective contrast sensitivity and visual satisfaction were not assessed. Further studies related to customized selection of aspheric IOLs should pay more attention to the full spectrum of preexisting corneal HOAs but not only SA [21]. Further attentions should also be paid to investigate how tilt, decentration and depth of focus impact on the optimization of optical quality in customized selection. It is also necessary to explore the differences in postoperative optical quality by OQAS among customized selection with different target ocular SA values. In addition, visual satisfaction questionnaire should be applied to complement with the objective optical quality assessments.

\section{Conclusions}

In summary, though customized selection of aspheric IOL implantation shows less postoperative ocular aberrations than random selection, they demonstrate similar visual acuity, simulated contrast visual acuity, intraocular scatter and optical quality by using an OQAS system. These results suggest that a customized selection of aspheric IOLs based on corneal SA with a target postoperative ocular SA of zero may not result in an overall optical quality improvement compared to the random selection. In other words, comparably desirable optical quality could be achieved by both customized selection and random selection of aspheric IOL implantation.

\section{Abbreviations \\ BCVA: Best corrected visual acuity; D: Diopters; HOAs: Higher-order aberrations; IOL: Intraocular lens; MTF: Modulation transfer function; OQAS: Optical quality analysis system; OSI: Objective scatter index; OV: Optical quality analysis system value; PD: Pupil diameter; PSF: Point spread function; SA: Spherical aberration; SD: Standard deviation; SR: Strehl ratio; t-HOA: Total higher-order aberration; UCVA: Uncorrected visual acuity}

\section{Acknowledgments}

The authors appreciate that Dr. Jing-Yun Wang who provided the linguistic assistance and scientific comments for this paper.

\section{Authors' contributions}

Concept and design (QQT, JL, CJL); data acquisition (JL, JT); data analysis/ interpretation (QQT, JL, $\mathrm{XL}, \mathrm{CJ} \mathrm{L})$; drafting of the manuscript (QQT, JL, CJL); critical revision of the manuscript (QQT, JL, XL, JT, CJL); statistical analysis (QQT, JL, XL); securing funding: (XL, CJL); supervision (QQT, CJL); All authors read and approved the final manuscript.

\section{Funding}

This work was supported partly by Key Research Project of Sichuan Health and Family Planning Commission (No. 18ZD022) and Research Project of Sichuan Health and Family Planning Commission (No. 17PJ529). The funders had no role in study design, data collection and analysis, decision to publish, or preparation of the manuscript.

Availability of data and materials

Available upon request from the corresponding author.

\section{Ethics approval and consent to participate}

This study followed the tenets of the Declaration of Helsinki and was approved by the Institutional Review Board of Affiliated Hospital of North Sichuan Medical College. Written informed consents were obtained from all participants.

\section{Consent for publication}

Not applicable.

\section{Competing interests}

The authors declare that they have no competing interests.

\section{Author details}

${ }^{1}$ Department of Ophthalmology, Affiliated Hospital of North Sichuan Medical College, 1 Mao Yuan Nan Road, Nanchong 637000, Sichuan, China.

${ }^{2}$ Department of Ophthalmology and Optometry, North Sichuan Medical College, 1 Mao Yuan Nan Road, Nanchong 637000, Sichuan, China.

Received: 4 April 2019 Accepted: 9 July 2019

Published online: 18 July 2019

\section{References}

1. Schuster AK, Tesarz J, Vossmerbaeumer U. Ocular wavefront analysis of aspheric compared with spherical monofocal intraocular lenses in cataract 
surgery: systematic review with metaanalysis. J Cataract Refract Surg. 2015 41(5):1088-97.

2. Beiko GH, Haigis W, Steinmueller A. Distribution of corneal spherical aberration in a comprehensive ophthalmology practice and whether keratometry can predict aberration values. J Cataract Refract Surg. 2007; 33(5):848-58.

3. Chang DH, Rocha KM. Intraocular lens optics and aberrations. Curr Opin Ophthalmol. 2016;27(4):298-303.

4. Diaz-Douton F, Benito A, Pujol J, Arjona M, Guell JL, Artal P. Comparison of the retinal image quality with a Hartmann-shack wavefront sensor and a double-pass instrument. Invest Ophthalmol Vis Sci. 2006;47(4):1710-6.

5. Martinez-Roda JA, Vilaseca M, Ondategui JC, Giner A, Burgos FJ, Cardona G, et al. Optical quality and intraocular scattering in a healthy young population. Clin Exp Optom. 2011;94(2):223-9.

6. Pan AP, Wang QM, Huang F, Huang JH, Bao FJ, Yu AY. Correlation among lens opacities classification system III grading, visual function index-14, pentacam nucleus staging, and objective scatter index for cataract assessment. Am J Ophthalmol. 2015;159(2):241-7.e2.

7. Chen T, Yu F, Lin H, Zhao Y, Chang P, Lin L, et al. Objective and subjective visual quality after implantation of all optic zone diffractive multifocal intraocular lenses: a prospective, case-control observational study. $\mathrm{Br}$ Ophthalmol. 2016;100(11):1530-5.

8. Park CW, Kim H, Joo CK. Assessment of optical quality at different contrast levels in Pseudophakic eyes. J Ophthalmol. 2016;2016:4247973.

9. Saad A, Saab M, Gatinel D. Repeatability of measurements with a doublepass system. J Cataract Refract Surg. 2010;36(1):28-33.

10. Xu CC, Xue T, Wang QM, Zhou YN, Huang JH, Yu AY. Repeatability and reproducibility of a double-pass optical quality analysis device. PLoS One. 2015:10(2):e0117587.

11. Solomon JD. Outcomes of corneal spherical aberration-guided cataract surgery measured by the OPD-scan. J Refract Surg (Thorofare, NJ : 1995). 2010;26(11):863-9.

12. Packer $M$, Fine $\mathbb{H}$, Hoffman RS. Aspheric intraocular lens selection based on corneal wavefront. J Refract Surg (Thorofare, NJ : 1995). 2009;25(1):12-20.

13. Al-Sayyari TM, Fawzy SM, Al-Saleh AA. Corneal spherical aberration and its impact on choosing an intraocular lens for cataract surgery. Saudi J Ophthalmol. 2014;28(4):274-80.

14. Cai FF, Zheng GY, Wang HJ, Li L, Liu Y. Clinical observation of visual quality after the individual implantation of intraocular lens guided by corneal $\mathrm{Q}$ value. Zhonghua Yan Ke Za Zhi. 2017:53(11):814-20.

15. Vilaseca M, Arjona M, Pujol J, Issolio L, Guell JL. Optical quality of foldable monofocal intraocular lenses before and after injection: comparative evaluation using a double-pass system. J Cataract Refract Surg. 2009;35(8): 1415-23.

16. Liao X, Lin J, Tian J, Wen B, Tan Q, Lan C. Evaluation of optical quality: ocular scattering and aberrations in eyes implanted with diffractive multifocal or Monofocal intraocular lenses. Curr Eye Res. 2018;43(6):696-701.

17. Kamiya K, Shimizu K, Igarashi A, Kobashi H, Ishii R, Sato N. Clinical evaluation of optical quality and intraocular scattering after posterior chamber phakic intraocular lens implantation. Invest Ophthalmol Vis Sci. 2012;53(6):3161-6.

18. Xiao XW, Hao J, Zhang H, Tian F. Optical quality of toric intraocular lens implantation in cataract surgery. Int J Ophthalmol. 2015;8(1):66-71.

19. Holladay JT, Piers PA, Koranyi G, van der Mooren M, Norrby NE. A new intraocular lens design to reduce spherical aberration of pseudophakic eyes. J Refract Surg (Thorofare, NJ : 1995). 2002;18(6):683-91.

20. Piers PA, Manzanera S, Prieto PM, Gorceix N, Artal P. Use of adaptive optics to determine the optimal ocular spherical aberration. J Cataract Refract Surg. 2007;33(10):1721-6.

21. Wang L, Koch DD. Custom optimization of intraocular lens asphericity. J Cataract Refract Surg. 2007;33(10):1713-20.

22. Jia LX, Li ZH. Clinical study of customized aspherical intraocular lens implants. Int J Ophthalmol. 2014;7(5):816-21.

23. Nochez Y, Favard A, Majzoub S, Pisella PJ. Measurement of corneal aberrations for customisation of intraocular lens asphericity: impact on quality of vision after micro-incision cataract surgery. Br J Ophthalmol. 2010; 94(4):440-4.

24. Assaf A, Kotb A. Ocular aberrations and visual performance with an aspheric single-piece intraocular lens: contralateral comparative study. J Cataract Refract Surg. 2010;36(9):1536-42.
25. Page TP, Whitman J. A stepwise approach for the management of capsular contraction syndrome in hinge-based accommodative intraocular lenses. Clin Ophthalmol (Auckland, NZ). 2016;10:1039-46.

26. Lee H, Lee K, Ahn JM, Kim EK, Sgrignoli B, Kim TI. Double-pass system assessing the optical quality of pseudophakic eyes. Optom Vis Sci. 2014; 91(4):437-43.

27. Perez GM, Manzanera S, Artal P. Impact of scattering and spherical aberration in contrast sensitivity. J Vis. 2009;9(3):19.1-0.

28. Chantra S, Pachimkul P, Naripthaphan P. Wavefront and ocular spherical aberration after implantation of different types of aspheric intraocular lenses based on corneal spherical aberration. J Med Assoc Thai. 2011;94(Suppl 2):S71-5.

29. Denoyer A, Denoyer L, Halfon J, Majzoub S, Pisella PJ. Comparative study of aspheric intraocular lenses with negative spherical aberration or no aberration. J Cataract Refract Surg. 2009;35(3):496-503.

30. Nochez Y, Majzoub S, Pisella PJ. Effect of residual ocular spherical aberration on objective and subjective quality of vision in pseudophakic eyes. J Cataract Refract Surg. 2011;37(6):1076-81.

31. Beiko GH. Personalized correction of spherical aberration in cataract surgery. J Cataract Refract Surg. 2007:33(8):1455-60.

32. Einighammer J, Oltrup T, Feudner E, Bende T, Jean B. Customized aspheric intraocular lenses calculated with real ray tracing. J Cataract Refract Surg. 2009:35(11):1984-94

\section{Publisher's Note}

Springer Nature remains neutral with regard to jurisdictional claims in published maps and institutional affiliations.

\section{Ready to submit your research? Choose BMC and benefit from:}

- fast, convenient online submission

- thorough peer review by experienced researchers in your field

- rapid publication on acceptance

- support for research data, including large and complex data types

- gold Open Access which fosters wider collaboration and increased citations

- maximum visibility for your research: over $100 \mathrm{M}$ website views per year

At BMC, research is always in progress.

Learn more biomedcentral.com/submissions 